
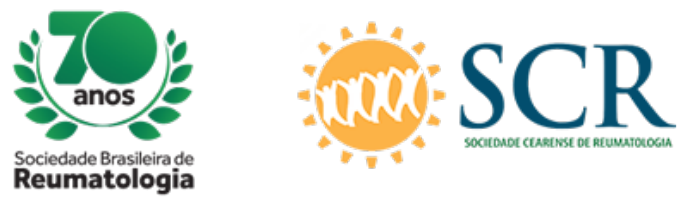

\title{
HYPERIMMUNOGLOBULIN D SYNDROME WITH COMPLETE RESPONSE TO ETANERCEPT: A REPORT OF
} TWO PEDIATRIC CASES.

Bruna de Lima Porto (Hospital de Clínicas de Porto Alegre, Porto Alegre, RS, Brasil), Afonso Papke (Hospital de Clínicas de Porto Alegre, Porto Alegre, RS, Brasil), Micheline Sulzbacher Batista (Hospital de

Clínicas de Porto Alegre, Porto Alegre, RS, Brasil), Ana Laura Fischer Kunzler (Hospital de Clínicas de Porto Alegre, Porto Alegre, RS, Brasil), Victoria Silveira de Carvalho (Hospital de Clínicas de Porto Alegre, Porto Alegre, RS, Brasil), Sandra Helena Machado (Hospital de Clínicas de Porto Alegre, Porto Alegre, RS, Brasil), Vanessa Hax (Hospital de Clínicas de Porto Alegre, Porto Alegre, RS, Brasil), Rafael Medonça da Silva Chakr (Hospital de Clínicas de Porto Alegre, Porto Alegre, RS, Brasil)

\section{BACKGROUND}

Hyperimmunoglobulin D syndrome (HIDS) is a rare autosomal-recessive disease characterized by fever attacks, accompanied by chills, headache, abdominal pain, and cervical lymphadenopathy. Individuals deficient in mevalonate kinase enzyme show a pro-inflammatory phenotype with large quantities of IgA, IgD, IL-1, IL-6 and TNF-alpha. Is usually characterized by an elevated serum polyclonal IgD, though normal levels do not exclude the disease.

Although HIDS drug treatment includes nonsteroidal anti-inflammatory drugs (NSAID), prednisone and biological medications, there is no consensus about how HIDS should be treated. Etanercept has been reported in a few cases with conflicting results. Herein, we report two cases of HIDS who treatment with Etanercept at the dose of $0.8 \mathrm{mg} / \mathrm{kg}$ once week resulted in the disappearance of febrile attacks and improvement of this patients' quality of life.

\section{CASE REPORT}

Case 1

A 2-year-old female patient with periodic fever since one month of age, accompanied by diffuse skin rash, oral ulcers, abdominal pain and loose stools. This patient was referred to a rheumatologist who requested genetic tests that showed genetic mutations in the MVK gene. With the established diagnosis of HIDS and without significant response to colchicine and anti-inflammatory drugs, we decided start biological therapy with etanercept. Patient has been using the medication for about 3 years with disease control and without serious adverse effects.

\section{Case 2}

A 3-year-oldmale patient presenting periodic fever since the age of 5 months, at regular intervals of about 30 days, accompanied by prostration, abdominal and joint pain. During the febrile episodes, cervical and abdominal lymph node enlargement was revealed on imaging tests and laboratory tests evidenced only increased inflammatory markers, without IgA or IgD increase. He presented partial improvement of the symptoms with NSAID. In view of typical symptoms (according to the Eurofever/PRINTO Clinical Classification Criteria) and the impossibility of performing genetic tests for financial reasons, a presumptive diagnosis of HIDS was made and etanercept started with excellent response. Patient has been using this medication for 7 months, remaining afebrile ever since.

\section{CONCLUSION}


Etanercept, a tumor necrosis factor (TNF) alpha receptor-immunoglobulin fusion protein, has been administered to some patients with HIDS with conflicting results. Within the therapeutic possibilities with biological therapy, TNF- $\alpha$ blockade has been reviewed only in descriptive retrospective studies, with a notable lack of head-to-head comparison against IL-1 blockade. Despite evidence scarcity, Etanercept seems to be an interesting therapeutic option in patients refractory to NSAID, as in the cases reported above. 